\title{
Transfer Operator-Based Extraction of Coherent Features on Surfaces
}

\author{
Kathrin Padberg-Gehle, Sebastian Reuther, Simon Praetorius, and Axel Voigt
}

\begin{abstract}
Transfer operator-based approaches have been successfully applied to the extraction of coherent features in flows. Transfer operators describe the evolution of densities under the action of the flow. They can be efficiently approximated within a set-oriented numerical framework and spectral properties of the resulting stochastic matrices are used to extract finite-time coherent sets. Also finite-time entropy, a density-based stretching quantity similar to finite-time Lyapunov exponents, is conveniently approximated by means of the discretized transfer operator. Transfer operator-based computational methods are purely probabilistic and derivative-free. Therefore, they can also be applied in settings where derivatives of the flow map are hardly accessible. In this paper, we summarize the theory and numerics behind the transfer operator approach and then introduce a straightforward extension, which allows us to study coherent structures in complex flows on triangulated surfaces. We illustrate our general computational framework with the well-known periodically driven double-gyre flow. To demonstrate the applicability of the approach for complex flows, we consider an approximation of the surface ocean flow, obtained by a numerical solution of the incompressible surface Navier-Stokes equation in a complicated geometry on the sphere.
\end{abstract}

\section{Introduction}

Transfer operator-based methods within a set-oriented numerical framework have only recently been recognized as powerful computational tools for analyzing and quantifying coherent structures and transport processes in time-dependent dynamical systems.

Of key interest are regions in the phase space of a nonautonomous dynamical system that remain coherent under the action of the flow. Almost-invariant sets $[2,6,8]$ are spatially fixed regions, while coherent sets $[7,10,11]$ move about with minimal dispersion. Almost-invariant and coherent sets can be efficiently identified

K. Padberg-Gehle $(\bowtie) \bullet S$. Reuther $\bullet$ S. Praetorius $\bullet$ A. Voigt

Institut für Wissenschaftliches Rechnen, TU Dresden, 01062 Dresden, Germany

e-mail: kathrin.padberg@tu-dresden.de; sebastian.reuther@tu-dresden.de;

simon.praetorius@tu-dresden.de; axel.voigt@tu-dresden.de 
via Perron-Frobenius operators (transfer operators). Recently, a unified functional analytic setting for optimal almost-invariant and coherent set constructions has been developed [10]. Transfer operators are linear Markov operators that can be approximated within a set-oriented framework [2]. Leading eigenvectors or singular vectors of the resulting stochastic matrices are heuristically used to determine the phase space structures of interest.

Transfer operators can also be employed to estimate finite-time expansive behavior along trajectories in autonomous and nonautonomous dynamical systems. Finite-time entropy (FTE) captures nonlinear stretching directly from the entropy growth experienced by a small localized density evolved by the transfer operator. In other words, FTE measures the increase in uncertainty of a small perturbation in the initial condition under the action of the flow and thus gives similar results to finite-time Lyapunov exponent calculations. Within the set-oriented approach an approximation of the FTE-field is obtained very efficiently and without relying on derivatives of the flow map. The FTE-concept has been introduced in [9], see also [18] for related previous work.

In this paper, we will sketch the theory and numerics behind the transfer operator approach. In addition, we will demonstrate how such probabilistic methods can be extended to highlight coherent features in complex flows approximated on unstructured grids. We begin by briefly reviewing the transfer operator framework in Sect. 2. In Sect. 3 the numerical approximation of such operators within a setoriented approach is described and as well as the extension to flows on triangulated surfaces. The discretized transfer operator is the fundamental tool for the extraction of coherent sets and transport barriers and we will introduce the respective approaches. In Sect. 4 we will illustrate these methods in two example systems. First, we will apply our computational framework to the well-known periodically driven double-gyre flow. Second, we consider an approximation of the global ocean surface flow obtained by the numerical solution of the incompressible surface Navier-Stokes equation in a complex geometry on the sphere [20] and highlight transport barriers and coherent sets. We will conclude with a short discussion and outlook in Sect. 5.

\section{Nonautonomous Dynamics, Transfer Operators and Transport}

We consider a nonautonomous differential equation

$$
\dot{\mathbf{x}}=\mathbf{u}(\mathbf{x}, t)
$$

with state $\mathbf{x} \in M \subset \mathbb{R}^{d}$, time $t \in \mathbb{R}$ and sufficiently smooth right-hand side $\mathbf{u}$ such that the flow map $\Phi(\mathbf{x}, t ; \tau): M \times \mathbb{R} \times \mathbb{R} \rightarrow M, M \subset \mathbb{R}^{d}$ exists; here $\tau$ denotes the flow time and $t$ the initial time. We are interested in extracting 
and visualizing coherent subsets of $M$, i.e. mobile regions in $M$ that minimally mix with the surrounding phase space. Coherent structures and their boundaries provide a time-dependent skeleton of the dynamics, similar to invariant manifolds of hyperbolic fixed points in autonomous dynamical systems.

Many approaches are based on finite-time Lyapunov exponents

$$
\Lambda_{\tau}(\mathbf{x}, t)=\frac{1}{2|\tau|} \log \left(\lambda_{\max }\left[D_{\mathbf{x}} \Phi(\mathbf{x}, t ; \tau)^{\top} D_{\mathbf{x}} \Phi(\mathbf{x}, t ; \tau)\right]\right) .
$$

Ridges in these scalar fields are frequently used to approximate transport barriers in the flow $[14,22]$. This often heuristically applied concept has been recently set on a sound mathematical basis [15]. Finite-time Lyapunov exponents have also been successfully used for the Lagrangian visualization of flow structures [12, 13].

Probabilistic approaches aim at a direct extraction of finite-time coherent sets by solving an optimization problem. To be more precise, one seeks to find pairs of sets $\left(A_{t}, A_{t+\tau}\right)$ such that

$$
\rho\left(A_{t}, A_{t+\tau}\right)=\frac{\mu\left(A_{t} \cap \Phi\left(A_{t+\tau}, t+\tau ;-\tau\right)\right)}{\mu\left(A_{t}\right)}
$$

is maximal under some constraints (conservation of mass, robustness with respect to perturbations). Such constraints are necessary to regularize the essentially ill-posed optimization problem [7]. Here $\mu$ is a reference probability measure on $M$ at time $t$. Equation (3) measures the proportion of the set $A_{t}$ at time $t$ that is mapped to the set $A_{t+\tau}$ at time $t+\tau$. One seeks to find sets such that $A_{t+\tau} \approx \Phi\left(A_{t}, t ; \tau\right)$. This problem can be solved by considering the Perron-Frobenius operator $\mathbf{P}_{t, \tau}: L^{1}(M, m) \rightarrow$ $L^{1}(M, m)$ associated with the flow map $\Phi$, where $m$ denotes Lebesgue measure. The transfer operator is defined by

$$
\mathbf{P}_{t, \tau} f(\mathbf{x})=\frac{f(\Phi(\mathbf{x}, t+\tau,-\tau))}{|\operatorname{det} D \Phi(\Phi(\mathbf{x}, t+\tau,-\tau), t, \tau)|}
$$

The interpretation is that if $f$ is a density and $f(\mathbf{x})$ the density value in $\mathbf{x}$ at time $t$, then $\mathbf{P}_{t, \tau} f(\mathbf{x})$ describes the density value in $\Phi(\mathbf{x}, t ; \tau)$ at time $t+\tau$ induced by the flow map. In $[7,10]$ it was shown that maximizing $\rho$ can be described in the framework of optimizing an inner product involving a compact self-adjoint operator obtained from $\mathbf{P}_{t, \tau}$. In order to avoid the technical functional analytic description underlying $[7,10]$, we will briefly recall the concept of finite-time coherent sets in the finitary setting in Sect. 3.2. This will be based on a finite-rank approximation of $\mathbf{P}_{t, \tau}$ in terms of a stochastic matrix, which will be introduced in Sect. 3.1 (see also [11]).

$\mathbf{P}_{t, \tau}$ may also be used to derive a stretching measure, termed finite-time entropy (FTE). It is similar to finite-time Lyapunov exponents and based on the concept of differential entropy $h(f)=-\int_{\Omega} f \log f d m$, where $\Omega$ is the support of the density $f$. Note that on a given domain $\Omega$, the uniform density maximizes $h$. 
For a given initial condition $\mathbf{x}_{0}$, let $f_{\epsilon, \mathbf{x}_{0}}:=\left(1 / m\left(B_{\epsilon}\left(\mathbf{x}_{0}\right)\right)\right) \mathbf{1}_{B_{\epsilon}\left(\mathbf{x}_{0}\right)}$ denote a uniform density supported on $B_{\epsilon}\left(\mathbf{x}_{0}\right)$, a ball of radius $\epsilon$ about $\mathbf{x}_{0}$. An $\epsilon$-smoothing operator is defined by $\mathbf{A}_{\epsilon} f(\mathbf{x}):=\left(1 / m\left(B_{\epsilon}(\mathbf{x})\right)\right) \int_{B_{\epsilon(\mathbf{x})}} f d m$.

The rate of increase in entropy experienced in the $\epsilon$-neighborhood of $\mathbf{x}_{0}$ over the time span $[t, t+\tau]$ of the $\epsilon$-perturbed dynamics can now be described by

$$
F T E_{\epsilon}\left(\mathbf{x}_{0}, t ; \tau\right):=\frac{1}{|\tau|}\left[h\left(\mathbf{A}_{\epsilon} \mathbf{P}_{t, \tau} f_{\epsilon, \mathbf{x}_{0}}\right)-h\left(f_{\epsilon, \mathbf{x}_{0}}\right)\right] .
$$

Note that $F T E_{\epsilon}$ takes high values when the evolved density $\mathbf{A}_{\epsilon} \mathbf{P}_{t, \tau} f_{\epsilon, \mathbf{x}_{0}}$ is very spread out. To be more precise, $F T E_{\epsilon}$ measures nonlinear stretching and the resulting quantity is very much determined by the sum of the positive finite-time Lyapunov exponents w.r.t $\mathbf{x}_{0}$. Thus, especially in the relevant setting of two-dimensional divergence-free flows, it can be well compared with finite-time Lyapunov exponents. In [9] several properties of $F T E_{\epsilon}$ and its deterministic $\operatorname{limit}_{\epsilon \rightarrow 0} F T E_{\epsilon}$ have been derived. In Sect. 3.3 we will outline a very efficient set-oriented approximation of the FTE-field.

\section{Set-Oriented Numerical Framework}

We now describe a set-oriented numerical framework for the approximation of the nonautonomous Perron-Frobenius operator in terms of a transition matrix of a finitestate Markov chain. The discretized transfer operator is the basis for extracting coherent sets (Sect.3.2) and for the computation of FTE-fields (Sect.3.3). In Sect. 3.4 we discuss how these approaches easily extend to the case of flows on a triangulated surface.

\subsection{Approximation of Transfer Operator}

Following [11], we consider some compact subset $X \subset M$ and a small neighborhood $Y$ of $\Phi(X, t ; \tau)$. Let $\left\{B_{1}, \ldots, B_{k}\right\}$ be a partition of $X,\left\{C_{1}, \ldots, C_{n}\right\}$ a partition of $Y$. The partition elements are typically generalized rectangles, but other settings are possible. Applying Ulam's method [23], a finite-rank approximation of $\mathbf{P}_{t, \tau}$ : $L^{1}(X, m) \rightarrow L^{1}(Y, m)$ is given via the transition matrix

$$
P_{i j}=\frac{m\left(B_{i} \cap \Phi\left(C_{j}, t+\tau ;-\tau\right)\right)}{m\left(B_{i}\right)}, i=1, \ldots, k, j=1, \ldots, n
$$


where we drop the $t$ and $\tau$-dependence of $P$ for brevity. In practice the entries $P_{i j}$ of the transition matrix $P$ are estimated via

$$
P_{i j} \approx \frac{\#\left\{r: \Phi\left(\mathbf{z}_{i, r}, t ; \tau\right) \in C_{j}\right\}}{R},
$$

with uniformly distributed sample points $\mathbf{z}_{i, r}, r=1, \ldots, R$ chosen in each partition element $B_{i}, i=1, \ldots, k$. So $P_{i j}$ describes the conditional probability that a point chosen at random in $B_{i}$ will be mapped to $C_{j}$ under the action of $\Phi(\cdot, t ; \tau)$. Note that $P$ is a sparse, row-stochastic matrix and thus all its eigenvalues are contained in the unit circle.

The interpretation of the $P$-induced dynamics is that if $\mathbf{p} \geq 0$ (componentwise) is a probability vector $\left(\sum_{i} p_{i}=1\right)$, then $\mathbf{p}^{\prime}=\mathbf{p} P$ is the push-forward of $\mathbf{p}$ under the discretized action of $\Phi(\cdot, t ; \tau)$. Note that the numerical scheme introduces diffusion—which is also theoretically needed for robust results [7, 10].

\subsection{Extracting Finite-Time Coherent Sets}

Consider a reference probability measure $\mu$ on $X$ at time $t$, which is discretely represented as a probability vector $\mathbf{p}$ with $p_{i}=\mu\left(B_{i}\right), i=1, \ldots, k$. The image probability vector on $Y$ at time $t+\tau$ is then simply computed as $\mathbf{q}=\mathbf{p} P$. We assume both $\mathbf{p}>0$ and $\mathbf{q}>0$ (component-wise) and form a normalized matrix $L$ via

$$
L_{i j}=\frac{p_{i} P_{i j}}{q_{j}} .
$$

This matrix has the property that $\mathbf{1}_{\mathbb{R}^{k}} L=\mathbf{1}_{\mathbb{R}^{n}}$. In [7, 11] it was shown that (under some technical assumptions) the problem of finding optimal coherent sets can be approximated by considering the left eigenvectors $\mathbf{w}_{2} \in \mathbb{R}^{k}$ of $L L^{*}$ and $\hat{\mathbf{w}}_{2} \in \mathbb{R}^{n}$ of $L^{*} L$ to the second largest eigenvalue $\lambda_{2}<1$. Here $L^{*}=P^{\top}$. Note that these two eigenvalue problems can be turned into the task of finding leading singular values and corresponding left and right singular vectors of a sparse matrix (see [11] for the exact construction), which can be very efficiently computed by iterative schemes (e.g. svds in MATLAB). The signed vector entries of $\mathbf{w}_{2}$ and $\hat{\mathbf{w}}_{2}$ can be interpreted as relaxations of indicator functions of the sets $A_{t}$ and $A_{t+\tau}$ and their complements. Thus the vector $\mathbf{w}_{2}$ defines fuzzy coherent sets on $X$, whereas $\hat{\mathbf{w}}_{2}$ represents their image on $Y$. Optimal partitions of $X$ and $Y$ into finite-time coherent pairs can be approximated via a line search in $\mathbf{w}_{2}$ and $\hat{\mathbf{w}}_{2}[10,11]$, but plotting these vectors will already give a good visual impression of the location of coherent sets. 


\subsection{Set-Oriented Computation of FTE}

In the discrete context, densities (which are central to the FTE-construction in Eq. (5)) are now represented by discrete probability measures $\mu$. Thus, the entropy of a probability vector $\mathbf{p}$ with $p_{i}=\mu\left(B_{i}\right), i=1, \ldots, k$, is simply $H(\mathbf{p})=-\sum_{i=1}^{n} p_{i} \log p_{i}$. Under the assumption that all partition elements $\left\{B_{1}, \ldots, B_{k}\right\}$ are of equal volume, let $\delta_{i}$ be an $k$-vector with 1 in $i^{\text {th }}$ position and 0 elsewhere. Then the discrete FTE of a partition set $B_{i}$ is given by

$$
F T E\left(B_{i}, t ; \tau\right)=\frac{1}{|\tau|} H\left(\delta_{i} P\right)=-\frac{1}{|\tau|} \sum_{j=1}^{n} P_{i j} \log P_{i j} .
$$

In case of differing box volumes the equation reads

$$
\operatorname{FTE}\left(B_{i}, t ; \tau\right)=-\frac{1}{|\tau|}\left(\sum_{j=1}^{n} P_{i j} \log \frac{P_{i j}}{m\left(C_{j}\right)}+\log m\left(B_{i}\right)\right) .
$$

In addition, one may obtain stretching rates for the backward-time dynamics (i.e. from $t+\tau$ to $t$ ) directly from the forward-time computation. For this consider $\tilde{P} \approx$ $\mathbf{P}_{t+\tau,-\tau}$ that approximates the backward-time evolution where

$$
\tilde{P}_{j i}=\frac{m\left(B_{i} \cap \Phi\left(C_{j}, t+\tau ;-\tau\right)\right)}{m\left(\Phi\left(C_{j}, t+\tau ;-\tau\right)\right)}=\frac{m\left(B_{i}\right) P_{i j}}{\sum_{i=1}^{k} m\left(B_{i}\right) P_{i j}},
$$

i.e. $\tilde{P}$ is computable directly from $P$ and $m\left(B_{i}\right), i=1, \ldots, k$. We denote the FTEfield obtained from $\tilde{P}$ by $\widetilde{F T E}$.

Note that once the transition matrix $P$ has been computed, FTE-fields (5) can be very quickly approximated by application of Eqs. (9) or (10). In particular, we do not require to explicitly push forward probability densities with $P$. This is in contrast to the related uncertainty estimation proposed in [19].

\subsection{Extension to Flows on Triangulated Surfaces}

In order to calculate coherent sets for flows on closed oriented 2-manifolds $\Gamma$, i.e. surfaces of co-dimension one embedded in $\mathbb{R}^{3}$, we consider a partition $\mathscr{S}_{h}(\Gamma)$ of $\Gamma$ that approximates the surface. We assume that $\mathscr{S}_{h}(\Gamma)$ is a conforming triangulation consisting of triangles, such that

$$
\Gamma_{h}:=\bigcup_{S \in \mathscr{S}_{h}(\Gamma)} S \subset \mathbb{R}^{3}
$$


forms a polytope, with $\mathbf{v} \in \Gamma$ for all vertices $\mathbf{v} \in \mathscr{S}_{h}(\Gamma)$. Additionally, we assume the surface $\Gamma$ to be fixed, so in the construction of the approximate transfer operator outlined in Sect. 3.1 one only needs to consider a single partition.

The transfer operator $\mathbf{P}_{t, \tau}: L^{1}(\Gamma, m) \rightarrow L^{1}(\Gamma, m)$ is approximated via

$$
P_{i j}=\frac{m\left(S_{i} \cap \Phi\left(S_{j}, t+\tau ;-\tau\right)\right)}{m\left(S_{i}\right)}
$$

where $S_{i}$ denotes the $i$-th triangle in the triangulation $\mathscr{S}_{h}(\Gamma)$ and $m$ Lebesgue measure on $\Gamma_{h}$.

The surface $\Gamma$ is assumed to be oriented, thus also its approximation $\Gamma_{h}$. This orientation is given by the local numbering of the vertices $\mathbf{v}^{S}$ of the triangles $S$ in $\mathscr{S}_{h}$. So one can calculate the outward normal $\mathbf{n}_{S}$ to each triangle $S \in \mathscr{S}_{h}(\Gamma)$, by

$$
\hat{\mathbf{n}}_{S}:=\left(\mathbf{v}_{1}^{S}-\mathbf{v}_{0}^{S}\right) \times\left(\mathbf{v}_{2}^{S}-\mathbf{v}_{0}^{S}\right), \quad \mathbf{n}_{S}=\frac{\hat{\mathbf{n}}_{S}}{\left\|\hat{\mathbf{n}}_{S}\right\|_{2}},
$$

where the area of the triangle is given by $m(S)=\frac{1}{2}\left\|\hat{\mathbf{n}}_{S}\right\|_{2}$.

A simple approach to define a normal in each vertex of $\Gamma_{h}$ that approximates the normal of $\Gamma$ in this point is a weighted averaging over normals of adjacent triangles. Let $\mathscr{S}(\mathbf{v}):=\left\{S \in \mathscr{S}_{h}(\Gamma): \mathbf{v} \in S\right\}$, where $\mathbf{v} \in S$ means that $\mathbf{v}$ is a vertex of $S$. The vertex normal $\mathbf{n}(\mathbf{v})$ is then given by

$$
\mathbf{n}(\mathbf{v}):=\frac{\sum_{S \in \mathscr{S}(\mathbf{v}) \frac{\mathbf{n}_{S}}{m(S)}}}{\sum_{S \in \mathscr{S}(v) \frac{1}{m(S)}}}
$$

In order to approximate the flow map $\Phi(S, \cdot, \cdot)$ that maps points in $S$ to points in another triangle $S^{\prime} \in \mathscr{S}_{h}$, we first map a point $\mathbf{x} \in \Gamma_{h}$ to a point $\hat{\mathbf{x}} \notin \Gamma_{h}$ and project $\hat{\mathbf{x}}$ back to the polytope.

Let $\hat{S}:=\operatorname{argmin}\left(\operatorname{dist}(\hat{\mathbf{x}}, S): S \in \mathscr{S}_{h}\right)$ be the triangle closest to $\hat{\mathbf{x}}$ and let

$$
\hat{\mathbf{y}}:=\operatorname{argmax}\left(\left\|\mathbf{y}_{i}\right\|_{2}: \mathbf{y}_{i}=\hat{\mathbf{x}}-\mathbf{v}_{i}^{\hat{S}}, i=0,1,2\right)
$$

be the longest edge connecting $\hat{\mathbf{x}}$ to the vertices of $\hat{S}$. Then we define the projection $\pi_{h}: \mathscr{U} \rightarrow \Gamma_{h}$, where $\mathscr{U}$ is a neighborhood of $\Gamma_{h}$, by

$$
\pi_{h}(\hat{\mathbf{x}}):=\hat{\mathbf{x}}-\mathbf{n}_{\hat{S}}\left(\mathbf{n}_{\hat{S}} \cdot \hat{\mathbf{y}}\right) \in \Gamma_{h} .
$$

In order to obtain an approximation $\Phi_{h}: \Gamma_{h} \times \mathbb{R} \times \mathbb{R} \rightarrow \Gamma_{h}$ for the flow map $\Phi: \Gamma \times \mathbb{R} \times \mathbb{R} \rightarrow \Gamma$ using the triangulated surface $\Gamma_{h}$ and the projection operator $\pi_{h}$, we have to construct a time integration scheme on $\Gamma_{h}$. With stepsize $\Delta t$ one step 
of the Euler scheme followed by projection is given by

$$
\hat{\Phi}_{h}\left(\mathbf{x}_{t}, t ; \Delta t\right)=\pi_{h}\left(\mathbf{x}_{t}+\Delta t \mathbf{u}\left(\mathbf{x}_{t}, t\right)\right),
$$

for the nonautonomous system $\dot{\mathbf{x}}=\mathbf{u}(\mathbf{x}, t)$, with a given velocity field $\mathbf{u}$ and initial condition $\mathbf{x}(t)=\mathbf{x}_{t}$. We note that the error in the projection step is negligible compared to the local truncation error of the Euler scheme. The approximate flow map $\Phi_{h}(\cdot, t, t+\tau)$ is then obtained in terms of the respective sequence of Euler steps $\hat{\Phi}_{h}$. Let $\mathbf{u}$ be only represented by discrete values at the vertices of $\Gamma_{h}$, then the evaluation of $\mathbf{u}(\mathbf{x}, t)$ is given by a linear combination of the vertex values. To be more precise, let $\mathbf{x}=\lambda_{0} \mathbf{v}_{0}^{S}+\lambda_{1} \mathbf{v}_{1}^{S}+\lambda_{2} \mathbf{v}_{2}^{S}$ be the representation of $\mathbf{x} \in \Gamma_{h}$ in barycentric coordinates (with $\lambda_{0}+\lambda_{1}+\lambda_{2}=1$ ), then

$$
\mathbf{u}(\mathbf{x}, t)=\lambda_{0} \mathbf{u}\left(\mathbf{v}_{0}^{S}, t\right)+\lambda_{1} \mathbf{u}\left(\mathbf{v}_{1}^{S}, t\right)+\lambda_{2} \mathbf{u}\left(\mathbf{v}_{2}^{S}, t\right)
$$

The Euler-based integration scheme described here is only of order one and should therefore only be seen as a simple means to compute an approximate flow on a triangulated surface. This will be sufficient for the purpose of demonstrating the application of the transfer operator approach in Sect. 4.2, where only a modest flow time is needed. For studies that require higher numerical accuracy and longer flow times more sophisticated integration schemes should be used.

\section{Examples}

\subsection{Double-Gyre Flow}

As a benchmark problem for analysing flow structures, we consider the timedependent system of differential equations $[8,22]$

$$
\begin{aligned}
& \dot{x}=-\pi A \sin (\pi f(x, t)) \cos (\pi y) \\
& \dot{y}=\pi A \cos (\pi f(x, t)) \sin (\pi y) \frac{d f}{d x}(x, t),
\end{aligned}
$$

where $f(x, t)=\delta \sin (\omega t) x^{2}+(1-2 \delta \sin (\omega t)) x$. We choose $A=1, \delta=0.25$, $\omega=2 \pi$ and obtain a one-periodic flow. The FTLE fields at $t=0$ for flow times $\tau=1$ and $\tau=2$ are shown in Fig. 1, highlighting parts of the stable manifold of a hyperbolic periodic orbit on the $x$-axis. This invariant manifold serves as a major transport barrier.

For the transfer operator approach we partition the domain $M=[0,2] \times[0,1]$ by $n=32768=2^{15}$ square boxes. We form matrices $P_{i}, i=1, \ldots, 5$ by integrating with a fourth-order Runge Kutta scheme with constant stepsize $h=0.01$ over the 

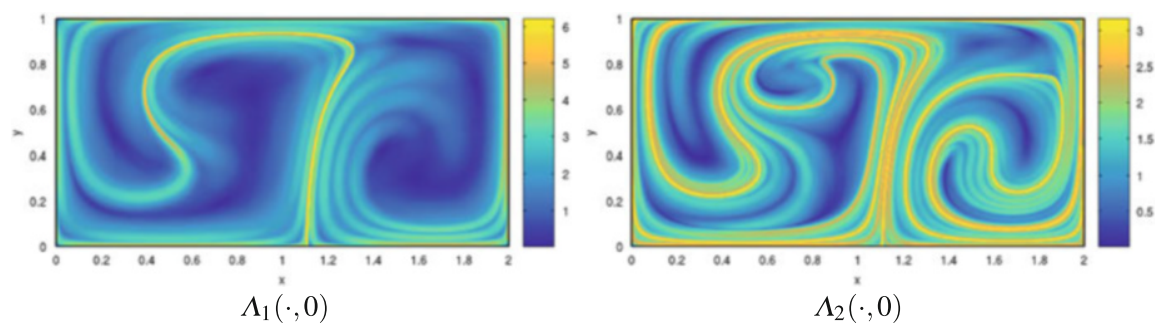

Fig. 1 FTLE fields on $[0,1]$ and on [0,2] highlight parts of the stable manifold of a hyperbolic periodic orbit

time intervals $I_{i}=[(i-1) \tau, i \tau], i=1, \ldots, 5$, where $\tau=0.2$, using $K=100$ uniformly distributed test points per box (inner grid points). The corresponding algorithms are implemented in the software package GAIO [3]. The product of matrices $Q=P_{1} P_{2} P_{3} P_{4} P_{5}$ approximates the transfer operator on the time interval $[0,1]$. As the flow is periodic, $Q^{2}$ does so on $[0,2]$. Note that we could also have approximated the transfer operator by forming a matrix directly over the interval $[0,1]$. However, again due to the periodicity, the concatenation of matrices allows us to easily change the intervals under consideration without any additional matrix approximations (which is the expensive part of the approach). Different FTE-fields extracted from the transition matrices are shown in Fig. 2 and they compare well with the FTLE fields in Fig. 1. We obtain the time-reversed FTE-fields $(\widetilde{F T E})$ by simple manipulation of the transition matrix according to Eq. (11). These pick up the unstable manifold emanating from the periodic orbit located on the upper boundary of $M$, see second row in Fig. 2.

We can also extract coherent sets via the vectors $\mathbf{w}_{2}$ and $\hat{\mathbf{w}}_{2}$ as described in Sect.3.2. Lebesgue measure on $M$ is taken as the reference measure. The corresponding coherent pairs are shown in Fig. 3. Here, negative and positive entries indicate the location of the two coherent pairs. Note that they align well with the transport barriers highlighted by FTLE or FTE.

Finally, we consider the stochastic differential equation (SDE)

$$
d \mathbf{X}_{t}=\mathbf{u}\left(\mathbf{X}_{t}, t\right) d t+\delta \mathbf{h}\left(\mathbf{X}_{t}, t\right) d \mathbf{W}_{t},
$$

with $\mathbf{u}$ being the right-hand side of $(13), \delta=0.001, \mathbf{h} \equiv(1,0)^{\top}$, and $\mathbf{W}$ denoting a Wiener process. We impose periodic boundary conditions and integrate the SDE on $[0,1]$ with an Euler-Maruyama scheme with stepsize $h=0.01$. The resulting paths are then used to set up the transition matrix as before-without any postprocessing. Again large FTE values are found in the vicinity of the major transport barrier (Fig. 4). 

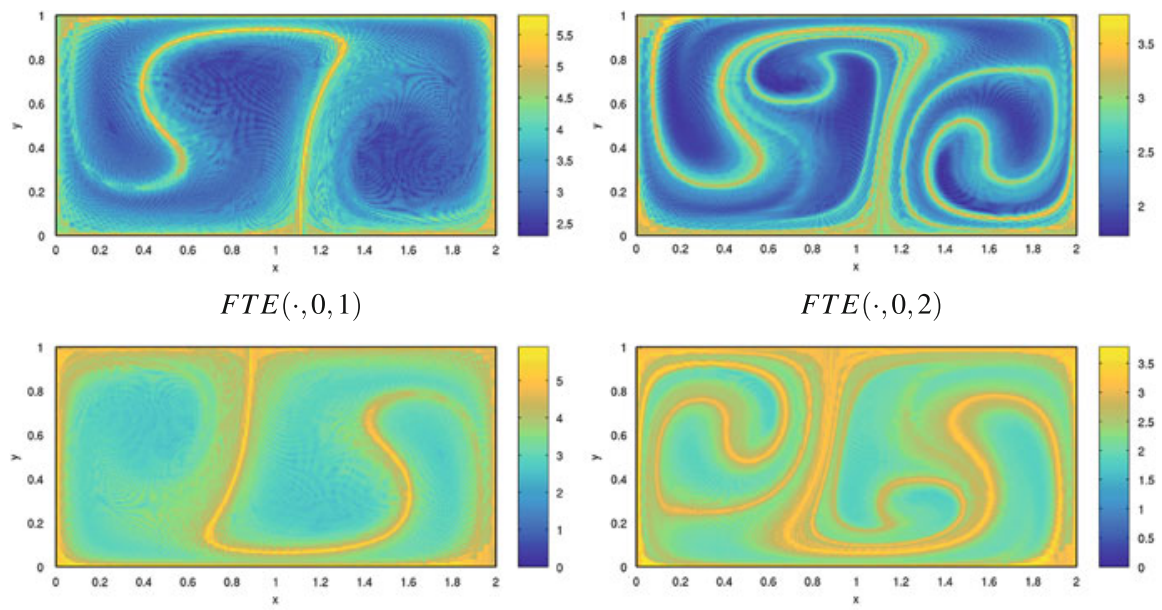

$\widetilde{F T E}(\cdot, 0,1)$

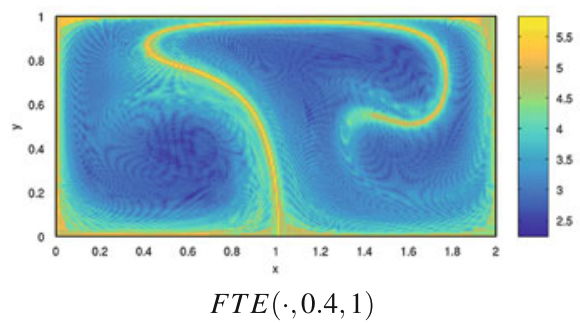

$$
\widetilde{F T E}(\cdot, 0,2)
$$

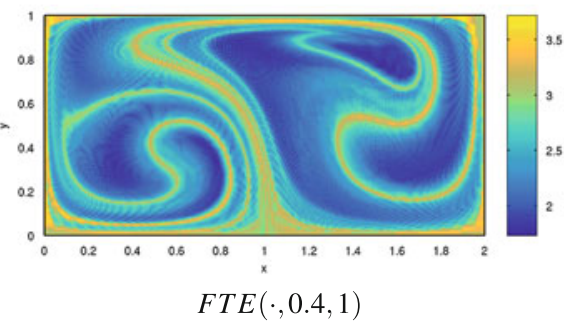

Fig. 2 Finite-time entropy fields computed on different intervals take high values in vicinity of major transport barriers
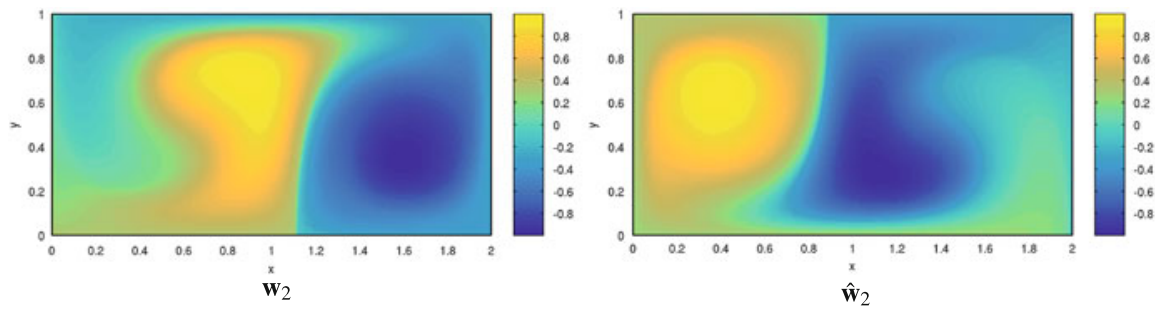

Fig. 3 Finite-time coherent sets on [0,1] are highlighted by the left and right second singular vectors $\mathbf{w}_{2}$ and $\hat{\mathbf{w}}_{2}$ of a reweighted transition matrix as described in Sect. 3.2

\subsection{Ocean Flow}

In [20] we have derived a simple two-dimensional model for fluid motion in the surface ocean, which is just driven by the Coriolis force. The basis is an incompressible surface Navier-Stokes equation with no-slip boundary conditions along 


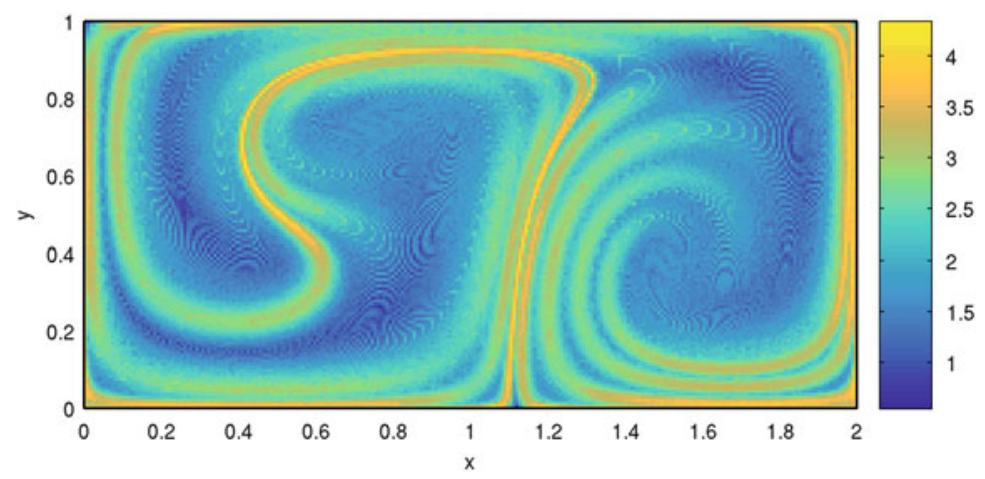

Fig. 4 Finite-time entropy field $\operatorname{FTE}(\cdot, 0,1)$ for the stochastic double gyre flow (14)

the continental borders. A diffuse domain approach [16] is used to describe the complex geometry on the whole spherical surface. The no-slip boundary condition is incorporated through a penalty like term. The equations now read

$$
\begin{aligned}
\partial_{t} \mathbf{u}+\mathbf{u} \cdot \nabla_{\Gamma} \mathbf{u}+\gamma \mathbf{n} \times \mathbf{u}+\frac{\mu}{\epsilon^{3}}(1-\xi) \mathbf{u} & =-\nabla_{\Gamma} p+\mu\left(\Delta_{\Gamma}^{R} \mathbf{u}+2 K \mathbf{u}\right) & & \text { on } \Gamma \\
\nabla_{\Gamma} \cdot \mathbf{u} & =0 & & \text { on } \Gamma
\end{aligned}
$$

with $\Gamma$ a two-dimensional surface, $\mathbf{u}$ the velocity vector, $p$ the pressure, $K$ the Gaussian curvature, $\nabla_{\Gamma}$ the surface gradient, $\Delta_{\Gamma}^{R}$ the Laplace-de Rham operator or Hodge-de Rham Laplacian, $\gamma=2 \omega \sin \theta$ the Coriolis parameter, $\omega$ the angular frequency, $\theta$ the latitude, $\mathbf{n}$ the surface normal, $\mu$ the viscosity, $\epsilon$ a small parameter, and $\xi$ a phase-field variable representing the continental and oceanic phases. For more details - also on the choice of parameters-we refer to e.g. [16, 17, 20]. Following [17], we can derive a convenient vorticity-stream function formulation, which reads

$\partial_{t} \phi+J(\psi, \phi+\gamma)-\frac{\mu}{\epsilon^{3}} \nabla_{\Gamma} \cdot\left((1-\xi) \nabla_{\Gamma} \psi\right)=\mu\left(\Delta_{\Gamma} \phi+2 \nabla_{\Gamma} \cdot\left(K \nabla_{\Gamma} \psi\right)\right) \quad$ on $\Gamma$

$$
\Delta_{\Gamma} \psi=\phi
$$

on $\Gamma$

where $\psi$ denotes the surface stream function, $\phi$ the surface vorticity, $J(\psi, \phi)=$ $\left(\mathbf{n} \times \nabla_{\Gamma} \psi\right) \cdot \nabla_{\Gamma} \phi$ the Jacobian and $\Delta_{\Gamma}$ the Laplace-Beltrami operator. In the special case of the two-dimensional $x$ - $y$-plane as the underlying geometry, the model agrees very well with common benchmark computations [21] and computations in multiply connected domains [1]. Note that the velocity field $\mathbf{u}$ can be retrieved from the stream function $\psi$ by the relation $\mathbf{u}=\nabla_{\Gamma} \psi \times \mathbf{n}$.

The system (15) is numerically solved using adaptive parametric finite elements $[4,5,24]$. Within the finite element toolbox AMDiS [24], we use a domain decom- 
position approach and a parallel iterative solver BiCGStab(ell). For all simulations the unit sphere $\mathbb{S}_{2} \subset \mathbb{R}^{3}$ serves as a simple representation of the earth. Moreover, an initial condition is chosen that includes the typical flow direction in the oceans to the west near the equator and to the east near the polar circles. The respective analytical form is given by $\psi_{0}(\mathbf{x})=\frac{1}{10} x_{3}\left(1-x_{3}^{2}\right)$ with $\mathbf{x}=\left(x_{1}, x_{2}, x_{3}\right)^{T} \in \Gamma=\mathbb{S}_{2} \subset \mathbb{R}^{3}$, which ignores the appearance of the continental borders. We therefore need an initialization phase to fulfill the internal boundary conditions.

The stream function that we obtain numerically as a solution of Eq. (15) turns out to be nearly stationary. For the sake of simplicity, we consider a stationary velocity field derived from a time average of the stream function for our further investigations. Figure 5 (top) shows the time averaged stream function in terms of streamlines as well as the respective flow direction. The major flow structuresespecially in the Indian, Pacific, Atlantic as well as in the Southern Ocean-can be observed and agree qualitatively with the known global circulation patterns.

To further visualize these major circulation patterns, we make use of the transfer operator-based approaches introduced in Sects. 2 and 3. As we have an autonomous velocity field, the coherent structures extracted by means of these methods should be aligned with streamlines and therefore our simplified setting is particularly suited for providing a proof of concept. We approximate the flow map numerically using the explicit Euler scheme (see Eq. (12)) with step size $\Delta t=0.1$ and flow time $\tau=50$ with respect to the approximated velocity field on the triangulated surface. The matrix entries $P_{i j}$ are estimated using 105 uniformly distributed test points per triangle $S_{i} \in \mathscr{S}_{h}$ and counting how many of them are mapped to $S_{j} \in \mathscr{S}_{h}, i, j=$ $1, \ldots, k$ with $k=50802$. Note that the complete triangulation of $\Gamma$ consists of 445812 triangles, but most of them are irrelevant for our probabilistic study (e.g. continents, highly resolved coastlines). We plot both $\operatorname{FTE}(\cdot, t ; \tau)$ and $\widetilde{F T E}(\cdot, t ; \tau)$ see Fig. 5 (bottom). In fact, the FTE-fields nicely highlight the boundaries of the major oceans and also the circulation patterns of the Antarctic Circumpolar Current, which are also clearly visible in the respective plots of the time averaged stream function (Fig. 5 (top)).

Using the same matrix $P$ from the FTE-study, we can also visualize finite-time coherent sets. For this, we approximate the leading left eigenvectors $\mathbf{w}_{i}, i=2,3, \ldots$ of $L L^{*}$ to eigenvalues very close to one, where we take normalized Lebesgue measure on $\Gamma_{h}$ as the reference probability measure and construct $L$ from $P$ as described in Sect. 3.2.

$\mathbf{w}_{2}$ indicates that the Northern and Southern Pacific make up two coherent structures (left panel of Fig. 6), $\mathbf{w}_{3}$ looks similar (not shown), whereas $\mathbf{w}_{4}$ (center panel) suggests a partition of the Atlantic Ocean into two coherent sets with the sharp boundary apparently nicely aligned both with streamlines and also with FTEridges when compared with Fig. 5 (center). Finally $\mathbf{w}_{5}$ (Fig. 6, right) highlights a coherent feature in the Indian Ocean, which is also clearly delineated by the separatrix visible in Fig. 5 (right). 

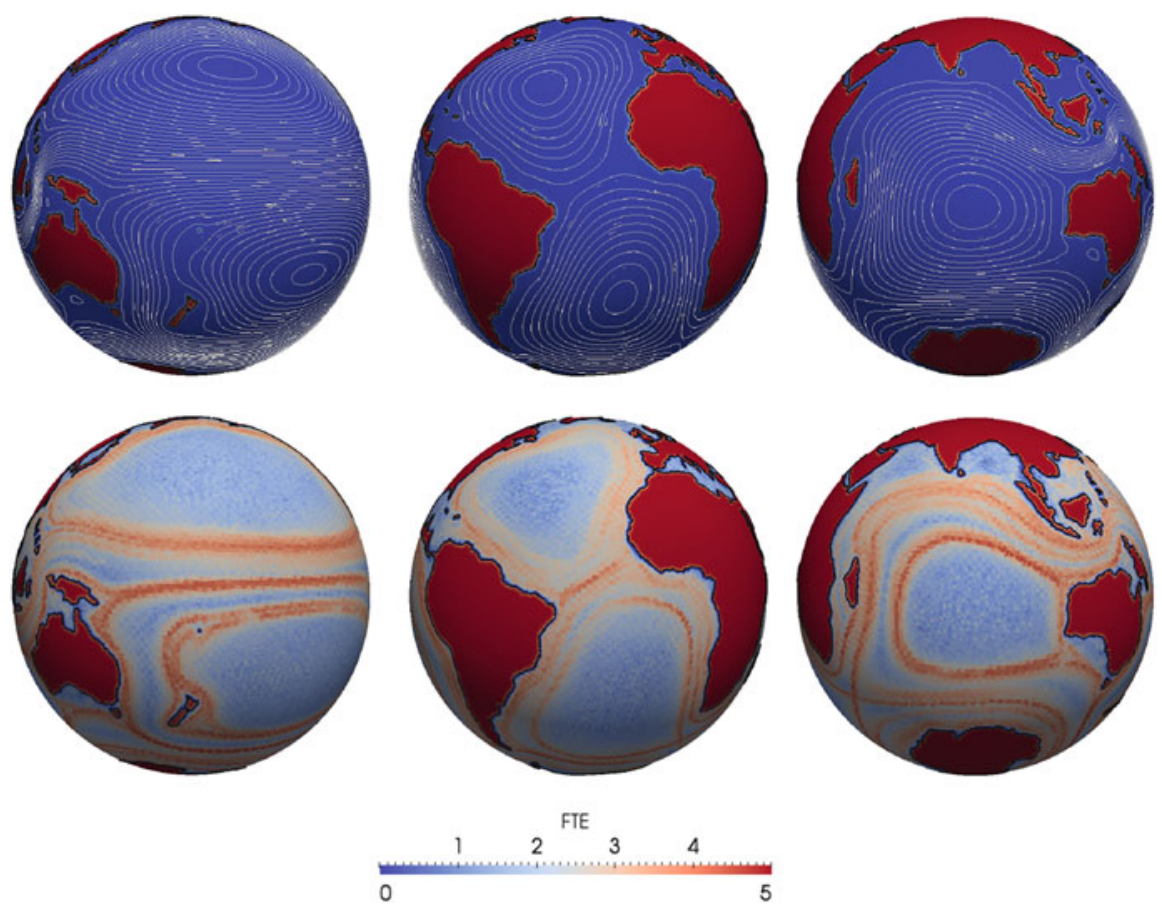

Fig. 5 Top: streamlines of the time averaged stream function indicate the major circulation patterns in the simulated ocean flow (from left to right: Pacific, Atlantic and Indian Ocean). Bottom: FTE is used to visualize the dynamical skeleton of the global ocean. Dark colors mark regions of high stretching indicating transport barriers. The results show the double-gyre structure in the Pacific Ocean delineated by regions of high FTE values (left), the circulation in the Indian Ocean (right), and the two major gyres in the Atlantic Ocean separated by a transport barrier (center)
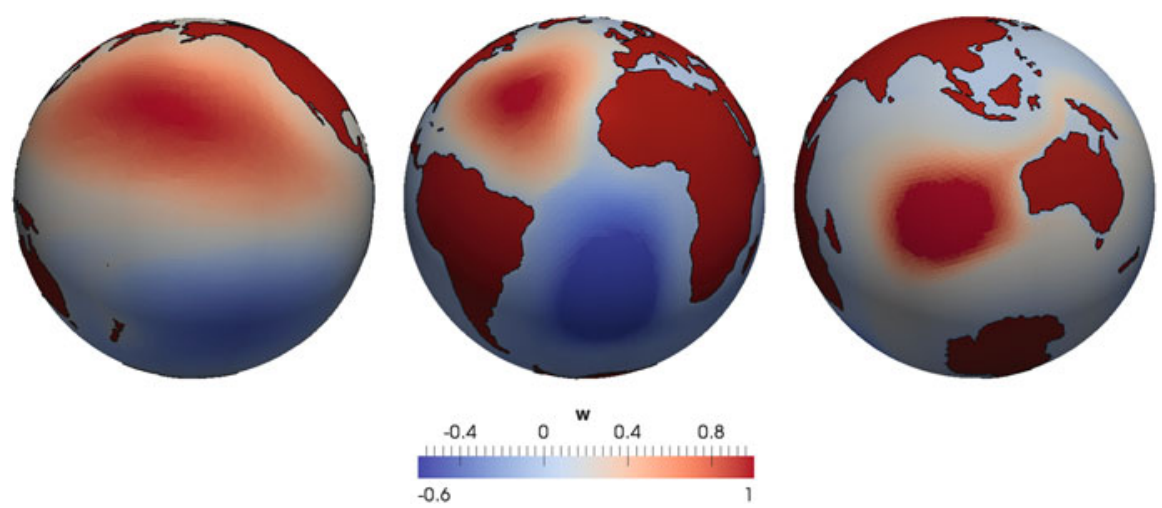

Fig. 6 Coherent structures in the simulated ocean flow are highlighted by the negative and positive entries of leading left eigenvectors $\mathbf{w}_{i}$ of a stochastic matrix derived from the transition matrix approximating the transfer operator of the flow map. Left: $\mathbf{w}_{2}$ defines coherent sets in the Pacific Ocean; center: $\mathbf{w}_{4}$ indicates a clear separation of the Northern and Southern Atlantic Ocean; right: $\mathbf{w}_{5}$ highlights a coherent feature in the Indian Ocean 


\section{Conclusion}

In this contribution we have briefly reviewed the transfer operator-based framework for the analysis of transport phenomena in time-dependent dynamical systems and the numerical approximation of transfer operators, finite-time coherent sets and transport barriers. Unlike the frequently used geometric approaches for the extraction of Lagrangian coherent structures [15], our framework is purely probabilistic and thus relies neither on smoothness properties of the flow map nor on any distance measure that might be difficult to approximate in a complex domain. Therefore, the transfer operator-based computational methods are easily applicable for studying the dynamical skeleton in flows confined to complicated geometries. In addition to a numerical study of the well-known double gyre flow, we have illustrated the strength of our approach in an example of a two-dimensional surface flow on a sphere $\mathbb{S}_{2} \subset \mathbb{R}^{3}$. With our methods we could highlight major circulation patterns of the global ocean. The spherical surface together with the continental borders give rise to a complex flow domain. Based on a transition matrix defined with respect to the triangulation of the surface and a stationary (numerically approximated) velocity field, we have visualized transport barriers and finite-time coherent sets. As expected, the extracted structures align well with equilines of the stream function in the considered autonomous dynamics, demonstrating that our approach returns meaningful results.

Future work will include the treatment of nonautonomous dynamics on evolving surfaces as well as the analysis of active fluid flows.

Acknowledgements KPG acknowledges funding from EU Marie-Skłodowska-Curie ITN Critical Transitions in Complex Systems (H2020-MSCA-2014-ITN 643073 CRITICS). SR and AV acknowledge support from DFG through Vo 899/6 and Vo 899/11. We further acknowledge the provided computing resources at ATLAS at ZIH at TU Dresden. Part of these results were obtained while AV was guest researcher at HIM at University Bonn.

\section{References}

1. Calhoun, D.: A Cartesian grid method for solving the two-dimensional streamfunctionvorticity equations in irregular regions. J. Comput. Phys. 176(2), 231-275 (2002)

2. Dellnitz, M., Junge, O.: On the approximation of complicated dynamical behavior. SIAM J. Numer. Anal. 36(2), 491-515 (1999)

3. Dellnitz, M., Froyland, G., Junge, O.: The algorithms behind GAIO - set oriented numerical methods for dynamical systems. In: Fiedler, B. (ed.) Ergodic Theory, Analysis, and Efficient Simulation of Dynamical Systems. Springer, Berlin (2009)

4. Dziuk, G., Elliott, C.M.: Finite elements on evolving surfaces. IMA J. Numer. Anal. 27(2), 231-275 (2007)

5. Dziuk, G., Elliott, C.M.: Surface finite elements for parabolic equations. J. Comput. Math. 25(4), 385 (2007)

6. Froyland, G.: Statistically optimal almost-invariant sets. Phys. D 200, 205-219 (2005) 
7. Froyland, G.: An analytic framework for identifying finite-time coherent sets in time-dependent dynamical systems. Phys. D 250, 1-19 (2010)

8. Froyland, G., Padberg, K.: Almost-invariant sets and invariant manifolds - connecting probabilistic and geometric descriptions of coherent structures in flows. Phys. D 238, 1507-1523 (2009)

9. Froyland, G., Padberg-Gehle, K.: Finite-time entropy: a probabilistic approach for measuring nonlinear stretching. Phys. D 241, 1612-1628 (2012)

10. Froyland, G., Padberg-Gehle, K.: Almost-invariant and finite-time coherent sets: directionality, duration, and diffusion. In: Bahsoun, G.F.W., Bose, C. (eds.) Ergodic Theory, Open Dynamics, and Coherent Structures, vol. 70, pp. 171-216. Springer, Berlin (2014)

11. Froyland, G., Santitissadeekorn, N., Monahan, A.: Transport in time-dependent dynamical systems: finite-time coherent sets. Chaos 20, 043,116 (2010)

12. Garth, C., Gerhardt, F., Tricoche, X., Hagen, H.: Efficient computation and visualization of coherent structures in fluid flow applications. IEEE Trans. Vis. Comput. Graph. 13(6), 14641471 (2007)

13. Garth, C., Wiebel, A., Tricoche, X., Joy, K.I., Scheuermann, G.: Lagrangian visualization of flow-embedded surface structures. Comput. Graph. Forum 27(3), 1007-1014 (2008)

14. Haller, G.: Distinguished material surfaces and coherent structures in three-dimensional fluid flows. Phys. D 149, 248-277 (2001)

15. Haller, G.: A variational theory of hyperbolic Lagrangian coherent structures. Phys. D 240, 574-598 (2011)

16. Li, X., Lowengrub, J., Rätz, A., Voigt, A.: Solving PDEs in complex geometries: a diffuse domain approach. Commun. Math. Sci. 7, 81-107 (2009)

17. Nitschke, I., Voigt, A., Wensch, J.: A finite element approach to incompressible two-phase flow on manifolds. J. Fluid Mech. 708, 418-438 (2012)

18. Padberg, K., Thiere, B., Preis, R., Dellnitz, M.: Local expansion concepts for detecting transport barriers in dynamical systems. Commun. Nonlinear Sci. Numer. Simul. 14(12), 4176-4190 (2009)

19. Reich, W., Scheuermann, G.: Analysis of streamline separation at infinity using time-discrete Markov chains. IEEE Trans. Vis. Comput. Graph. 18(12), 2140-2148 (2012)

20. Reuther, S.: Modellierung und Simulation von Oberflächenzirkulationen in Ozeanen (diploma thesis). Technical Report, Technische Universität Dresden (2012)

21. Schäfer, M., Turek, J.: Benchmark computations of laminar flow around a cylinder. In: Hirschel, B. (ed.) Flow Simulation with High-Performance Computers II. Springer, Berlin (1996)

22. Shadden, S.C., Lekien, F., Marsden, J.E.: Definition and properties of Lagrangian coherent structures from finite-time Lyapunov exponents in two-dimensional aperiodic flows. Phys. D 212, 271-304 (2005)

23. Ulam, S.: Problems in Modern Mathematics. Interscience, New York (1964)

24. Vey, S., Voigt, A.: AMDiS: adaptive multidimensional simulations. Comput. Vis. Sci. 10(1), 57-67 (2007) 